\title{
Hydrodynamic interaction with an array of porous circular cylinders
}

\author{
Min-Su Park, Weoncheol Koo, Yoonrak Choi \\ School of Naval Architecture and Ocean Engineering, University of Ulsan, Korea
}

\begin{abstract}
In the present study, the wave excitation forces acting on an array of porous circular cylinders are examined based on diffraction problems. To calculate the wave forces, the fluid domain is divided into three regions i.e. a single exterior region, $N$ interior regions and $N$ beneath regions, and the diffraction in each fluid region is expressed by an eigenfunction expansion method with using 3-dimension liner potential theory (Williams and Li, 2000). Especially, the present method is extended to the case of an array of truncated porous circular cylinders to calculate the heave forces as well as surge and sway forces. To verify this method, the numerical results obtained by eigenfunction are compared with these results obtained by higher order boundary element method (Choi et al., 2000). The numerical results obtained by this study are in good agreement with those results. By changing the numbers of porous circular cylinders, the angle of incident wave and the porosity rate of circular cylinders, the wave excitation forces such as surge, sway and heave on an array of truncated porous circular cylinders are investigated.
\end{abstract}

KEY WORDS: Wave excitation forces; Diffraction Problems; Eigenfunction expansion method; Truncated porous circular cylinder.

\section{INTRODUCTION}

It is well recognized that the wave diffraction problem about a vertical circular cylinder carried out by MacCamy and Fuchs (1954) is a typical problem with exact analytical solution in ocean engineering. As regarding shallow water wave diffraction around a vertical cylinder, Isaacson (1977, 1978) was one of the pioneers to derive analytical solutions. Under the assumptions of potential flow and linear wave theory, Spring and Monkmeyer (1974) have proposed a semianalytical solution obtained by an eigenfunction expansion approach firstly for impermeable cylinders and latter it has been simplified by Linton and Evans (1990) for $N$ bottommounted circular cylinders. Kagemoto and Yue (1986) have developed another solution that is formally exact within the context of the linear theory. They have shown how a general 3-dimensionalwater-wave diffraction problem concerning a structure consisting of a number of separated elements can be solved exactly in terms of the diffraction characteristics on each of the individual elements. In the case where the cylinder spacing is large relative to the incident wave length, approximate techniques may reasonably be used to quantify the hydrodynamic interactions between the members of multi-column structures. A popular approach, based on the

Corresponding author: Min-su Park

E-mail:mspark77@ulsan.ac.kr wide-spacing assumption is the so-called modified planewave approach firstly developed by McIver and Evans (1984), and later used in a number of applications by McIver (1984), Williams and Demirbilek (1988), Williams and Abul-Azm (1989), and Williams and Rangappa (1994). All of the above studies, however, assume that the cylinders are impermeable.

In case of porous cylinders, the wave motions in the exterior and all interior fluid regions are expressed by Williams and Li $(1998,2000)$. They had divided the fluid domain into $N+1$ regions, a single exterior region and $N$ interior regions. The diffraction potential in each fluid region was expressed with an eigenfuncition expansion method. Wang and Ren(1994) were the earliest to study the wave interaction with a concentric surface piercing porous outer cylinder protecting an impermeable inner cylinder. The free surface elevation, net hydrodynamic forces and wave induced over turning moments on both cylinders were determined analytically. It was reported that the outer porous cylinder is significantly effective to reduce the hydrodynamic force and wave run-up on the inner cylinder compared to be exposed to direct wave impact.

In the present study, the numerical analysis method is developed with eigenfunction expansion approach method which is expressed by Williams and $\mathrm{Li}$ (2000). The analysis method could be applied for the wave force evaluation to any cases such as for the array of impermeable or permeable circular cylinders. Especially, the present method could be extended to the case of an array of truncated porous circular 
cylinders to calculate the heave forces as well as surge and sway forces. Firstly, the results obtained from the present method are compared with the results obtained from HOBEM (Choi et al., 2000) in order to verify the developed numerical analysis method. Moreover, the wave force and wave run-up acting on the various cylinders are presented, and the comparison between the impermeable cylinder and the permeable cylinder is made for the wave force and wave runup in order to examine the effects of porosity. From these results, it is suggested that the present method is very useful to evaluate the wave force acting on the array of impermeable or permeable truncated vertical circular cylinders. It is found that the porosity of cylinders is remarkably effective to reduce the wave excitation forces and wave run-up.

\section{MATHEMATICAL FORMULATION}

It is formulated by the complete boundary-value problem in the general diffraction theory as follows. The basic flow is assumed to be oscillatory, incompressible, and irrotational so that the fluid velocity may be represented as the gradient of a scalar potential, $\Phi$. Under potential theory, the total velocity potential, $\Phi$, is obtained by a sum of the incident and a scattered potential. An arbitrary array of $N$ porous circular cylinders is situated in water of uniform depth $d$ and the clearance beneath each cylinder is denoted by $h$. The radius of the $j$ th cylinder is $a_{j}$ and the global Cartesian coordinate system $(x, y, z)$ is defined with an origin located on the stillwater level with the $z$-axis directed vertically upwards. The center of each cylinder at $\left(x_{j}, y_{j}\right)$ is taken as the origin of a local polar coordinate system $\left(r_{j}, \theta_{j}\right)$, where $\theta_{j}$ is measured counterclockwise from the positive $x$-axis. The center of the $k$ th cylinder has polar coordinates $\left(R_{j k}, \alpha_{j k}\right)$ relative to the $j$ th cylinder. The coordinate relationship between the $j$ th and $k$ th cylinders is shown in Fig. 1.Moreover, the fluid domain is divided into three regions i.e. a single exterior region, $N$ interior regions and $N$ beneath regions.
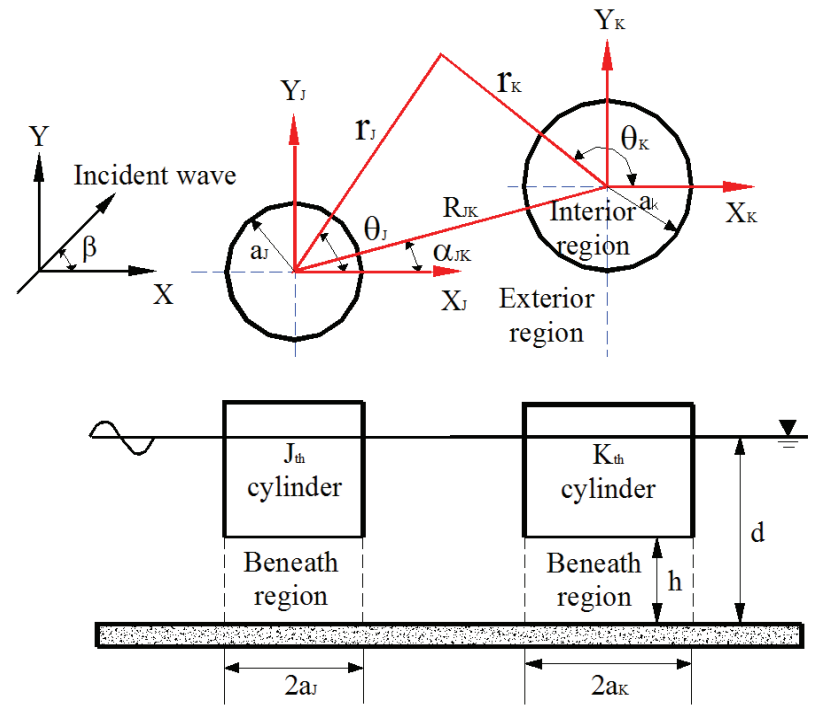

Fig. 1 Coordinate system for an array of porous cylinders.
The array of the cylindrical structure is subjected to a train of regular waves of height $H$ and angular frequency $\omega$ propagating at an angle $\beta$ to the positive $x$-axis. For the uniform geometry of the array structure, the depth dependence of the problem can be written as follow,

$\Phi(x, y, z, t)=R_{e}\left[\varphi(x, y) f(z) e^{-i \omega t}\right]$

where $\operatorname{Re}[]$ denotes the real part of a complex expression and

$f(z)=-\frac{i g(H / 2) \cosh k(z+d)}{\omega \cosh k d}$

in which $g$ is the acceleration of gravity and the wave number $k$ is the positive real root of the dispersion relation, $\omega^{2}=g k \tanh k d$.

The incident plane wave potential can be expressed with the $j$ th local polar coordinate system by

$\Phi_{i n}^{j}=I_{j} e^{i k r_{j} \cos \left(\theta_{j}-\beta\right)}$

where $I_{j}\left(=e^{i k(x j \cos \beta+y j \sin \beta}\right)$ is a phase factor associated with cylinder $j$. This equation can be written as follow,

$\Phi_{i n}^{j}=I_{j} \sum_{n \rightarrow-\infty}^{\infty} J_{n}\left(k r_{j}\right) e^{i n\left(\pi / 2-\theta_{j}+\beta\right)}$

in which $J_{n}$ denotes the Bessel function of the first kind of order $n$.

Following boundary conditions such as the Helmholtz equation and the usual radiation, the general form for the scattered wave emanating from cylinder $j$ can be written as follow,

$\Phi_{s}^{j}=\sum_{n \rightarrow-\infty}^{\infty} A_{n}^{j} Z_{n}^{j} H_{n}\left(k r_{j}\right) e^{i n \theta_{j}}$

where $A_{n}^{j}$ are the unknown complex potential coefficients, and the factors $Z_{n}^{j}$ can be determined by the boundary conditions on the cylinder surface, where for the limiting case of cylinders being rigid leads to,

$Z_{n}^{j}\left(=Z_{-n}^{j}\right)=\frac{J_{n}^{\prime}\left(k a_{j}\right)}{H_{n}^{\prime}\left(k a_{j}\right)}$

here $H_{n}$ is the Hankel function of first kind of order $n$ and $J_{n}^{\prime}$ and $H_{n}$ are the first derivatives of the Bessel and Hankel function of the first kind respectively.

The total potential,which is obtained by a sum of the incident and a scattered potential,in the exterior region can therefore be written as follow, 


$$
\Phi_{I}=\Phi_{i n}+\sum_{j=1}^{N} \Phi_{s}^{j}=e^{i k r \cos (\theta-\beta)}+\sum_{j=1}^{N} \sum_{n=-\infty}^{\infty} A_{n}^{j} Z_{n}^{j} H_{n}\left(k r_{j}\right) e^{i n \theta_{j}}
$$

To account for interaction among the cylinders, it is necessary to evaluate the scattered potential $\Phi_{s}^{\lambda}$ in terms of the representation of the incident potential $\Phi_{I}^{\lambda}$ at cylinder $j$, $j=1,2,3, \ldots, N, j \neq \lambda$. This can be accomplished by using Graf's addition theorem for Bessel functions to give,

$$
H_{n}\left(k r_{\lambda}\right) e^{i n\left(\theta_{\lambda}-\alpha_{\lambda j}\right)}=\sum_{m=-\infty}^{\infty} H_{n+m}\left(k R_{\lambda j}\right) J_{m}\left(k r_{j}\right) e^{i m\left(\pi-\alpha_{\lambda j}-\theta_{j}\right)}
$$

for $\lambda, j=1,2,3, \ldots, N, j \neq \lambda$ (Abramowitz and Stegun, 1972). Equation (8) is valid for $r_{j}<R_{\lambda j}$, which is true on the boundary of the $j$ th cylinder for all $\lambda$. The exterior region potential can be expressed as follows,

$$
\begin{aligned}
& \Phi_{1}^{j}\left(r_{j}, \theta_{j}\right) \\
& =\sum_{n=-\infty}^{\infty}\left[I_{j} J_{n}\left(k r_{j}\right) e^{i n\left(\pi / 2-\beta-\theta_{j}\right)}+A_{n}^{j} Z_{n}^{j} H_{n}\left(k r_{j}\right) e^{i n \theta_{j}}\right] \\
& +\sum_{\substack{\lambda=1 \\
\lambda \neq j}}^{N} \sum_{\substack{n=-\infty \\
n}}^{\infty} A_{n}^{\lambda} Z_{n}^{\lambda} \sum_{m=-\infty}^{\infty} J_{m}\left(k r_{j}\right) H_{n+m}\left(k R_{\lambda j}\right) e^{i m\left(\pi / 2-\theta_{j}+\alpha_{\lambda j}\right)+i n \alpha_{\lambda j}}
\end{aligned}
$$

in which if $r_{j}<R_{\lambda j}$ for all $\lambda$, i.e., this expansion is valid near cylinder $j$. The final term in equation (9) may be rearranged as follow,

$$
\begin{aligned}
& \Phi_{1}^{j}\left(r_{j}, \theta_{j}\right)=\sum_{n=-\infty}^{\infty}\left\{A_{n}^{j} Z_{n}^{j} H_{n}\left(k r_{j}\right)+\right. \\
& \left.\left[I_{j} e^{i n(\pi / 2-\beta)}+\sum_{\substack{\lambda=1 \\
\lambda \neq j}}^{N} \sum_{m=-\infty}^{\infty} A_{m}^{\lambda} Z_{m}^{\lambda} H_{m-n}\left(k R_{\lambda j}\right) e^{i(m-n) \alpha_{\lambda j}}\right] J_{n}\left(k r_{j}\right)\right\} e^{i n \theta_{j}}
\end{aligned}
$$

By the way, if the structure has some pores, it would be treated with the fluid flow through the pore. The wall of each cylinder is assumed to be thin with fine pores. The fluid flow passing through the porous walls is assumed to obey Darcy's law. Hence, the porous flow velocity is linearly proportional to the pressure difference across the thickness of the porous cylinder. Now the hydrodynamic pressure $p(x, y, z, t)=\operatorname{Re}\{p(x$, y) $\left.f(z) e^{-i \omega t}\right\}$ at any point in the fluid domain may be determined from the linearized Bernoulli equation as $P(x, y)=\rho i \omega \Phi(x, y)$ where $\rho$ is the fluid density. Therefore it follows that (Williams and $\mathrm{Li}, 2000$ )

$\frac{\partial \Phi_{2}^{(j)}}{\partial r}=\frac{\gamma}{\mu} \rho i \omega\left[\Phi_{2}^{(j)}-\Phi_{1}\right] \quad$ on $\quad r_{j}=a_{j} \quad j=1,2,3, \ldots, N$

where $\mu$ is the coefficient of dynamic viscosity and $\gamma$ is a material constant having the dimension of length. Subsequently, the porosity of the cylinder will be characterized by the dimensionless parameter, $G$. The body boundary condition on the porous cylinder can be expressed with the $G$ as follow,

$$
\frac{\partial \Phi_{2}^{(j)}}{\partial r}=i G\left[\Phi_{2}^{(j)}-\Phi_{1}\right]
$$

in which, $G=\frac{\rho \omega \gamma}{\mu}$

Also, the porosity parameter $G$, which is obtained from the numerical analysis and the numerical model experiment, can be expressed as follows (Cho, 2003),

$G=9.172 P-0.155$

where $P$ is the porosity rate of cylinder.

Following the Helmholtz equation, the potential in the $j$ th interior region, $\Phi_{2}{ }^{j}$, can be written as follow,

$$
\Phi_{2}^{j}=\sum_{n=-\infty}^{\infty} B_{n}^{j} J_{n}\left(k r_{j}\right) e^{i n \theta_{j}} \quad j=1, \ldots, N
$$

for $j=1,2, \ldots, N$, where $B_{n}^{j}$ are unknown potential coefficients. Applying boundary conditions, equation (5) and (7) by means of the properties of eigenfunctions, leads to following relationship between the potential coefficients $A_{n}^{j}$ and $B_{n}^{j}$,

$$
I_{j}(i)^{n} e^{-i n \beta}+\sum_{\substack{\lambda=1 \\ \lambda \neq j}}^{N} \sum_{m=-\infty}^{m=\infty} A_{m}^{\lambda} Z_{m}^{\lambda} H_{m-n}\left(k R_{\lambda j}\right) e^{i(m-n) \alpha_{\gamma j}}=B_{n}^{j}-A_{n}^{j}
$$

$$
I_{j}(i)^{n} e^{-i n \beta} J_{n}\left(k a_{j}\right)+A_{n}^{j} Z_{n}^{j} H_{n}\left(k a_{j}\right)
$$$$
+\sum_{\substack{\lambda=1 \\ \lambda \neq j}}^{N} \sum_{m=-\infty}^{m=\infty} A_{m}^{\lambda} Z_{m}^{\lambda} H_{m-n}\left(k R_{\lambda j}\right) e^{i(m-n) \alpha_{\gamma j}} J_{n}\left(k a_{\lambda j}\right)
$$$$
=B_{n}^{j}\left[J_{n}\left(k a_{j}\right)+\frac{i J_{n}^{\prime}\left(k a_{j}\right)}{G}\right] j=1,2, \ldots, N
$$

Combining equation (15) and (16), and using the Wronskian relationship for the Bessel functions results in the following infinite systems of equations,

$$
\begin{gathered}
A_{n}^{j}\left[1+\frac{2 G}{\pi k a_{j} J_{n}^{\prime}\left(k a_{j}\right) H_{n}^{\prime}\left(k a_{j}\right)}\right] \\
+\sum_{\substack{\lambda=1 \\
\lambda \neq j}}^{N=\infty} \sum_{\substack{m=-\infty \\
m=-\infty}}^{\lambda} Z_{m}^{\lambda} H_{m-n}\left(k R_{\lambda j}\right) e^{i(m-n) \alpha_{\lambda j}}=-I_{j}(i)^{n} e^{-i n \beta} \\
j=1,2, \ldots, N,-\infty<n<\infty
\end{gathered}
$$

$$
B_{n}^{j}=-\frac{2 G}{\pi k a_{j}} \times \frac{A_{n}^{j}}{H_{n}^{\prime}\left(k a_{j}\right) J_{n}^{\prime}\left(k a_{j}\right)}
$$


In order to calculate the potential coefficients $A_{n}^{j}$ the infinite system in equation (17) and (18) is truncated to a $(2 M+1) N$ system to equation in $(2 M+1) N$ unknowns,

$$
\begin{gathered}
I_{j}(i)^{n} e^{-i n \beta}+\sum_{\substack{\lambda=1 \\
\lambda \neq j}}^{N} \sum_{m=-M}^{m=M} A_{m}^{\lambda} Z_{m}^{\lambda} H_{m-n}\left(k R_{\lambda j}\right) e^{i(m-n) \alpha_{\lambda j}} \\
=-A_{n}^{j}\left[1+\frac{2 G}{\pi k a_{j} J_{n}^{\prime}\left(k a_{j}\right) H_{n}^{\prime}\left(k a_{j}\right)}\right] \\
j=1,2, \ldots, N,-\infty<n<\infty
\end{gathered}
$$

Good accuracy can be achieved by increasing $M$ in spite of the expense of computing time. Except for the cylinders being very close together, taking $M=10$ could achieve accurate results to all examinations. The potential coefficients $B_{n}^{j}$ may then be obtained from equation (18). The velocity potentials in each fluid region may be determined in the same manner.

The solution to a number of limiting cases may be obtained from equation (19). If the porosity parameter $G=0$ (which corresponds to an impermeable cylinder), the linear system in equation (19) becomes

$$
\begin{gathered}
I_{j}(i)^{n} e^{-i n \beta}+\sum_{\substack{\lambda=1 \\
\lambda \neq j}}^{N} \sum_{m=-M}^{m=M} A_{m}^{\lambda} Z_{m}^{\lambda} H_{m-n}\left(k R_{\lambda j}\right) e^{i(m-n) \alpha_{\lambda j}}=-A_{n}^{j} \\
j=1,2, \ldots, N,-\infty<n<\infty
\end{gathered}
$$

which is similar to the result of Linton and Evans (1990).

Using the method of separation of variables, a physically acceptable general solution for the beneath region can be constructed as follow,

$\varphi_{b}^{j}=\sum_{n=0}^{\infty}\left[\frac{C_{n 0}}{2}\left(\frac{r}{a}\right)^{n}+\sum_{m=1}^{\infty} C_{n m} \frac{I_{n}(m \pi r / h)}{I_{n}\left(m \pi a_{j} / h\right)} \cos (m \pi(z+d) / h)\right]$

$\cos n \theta$

Valid for $-d \leq z \leq-(d-h)$ and $r \leq a$; where $C_{n m}(m=0,1,2, \ldots)$ are arbitrary constants. Here $I_{n}(m \pi r / h)$ is the modified Bessel function of first kind of order $n$. Let us define $\zeta_{b}^{j}(r, z)$ as follows,

$$
\begin{aligned}
& \varsigma_{b}^{j}(r, z)=\frac{C_{n 0}}{2}\left(\frac{r}{a}\right)^{n} \\
& +\sum_{m=1}^{\infty} C_{n m} \frac{I_{n}(m \pi r / h)}{I_{n}\left(m \pi a_{j} / h\right)} \cos (m \pi(z+d) / h)
\end{aligned}
$$

such that at $r=a$, it becomes a half range Fourier cosine series expansion $\varsigma_{b}^{j}(a, z)=\frac{C_{n 0}}{2}+\sum_{m=1}^{\infty} C_{n m} \cos (m \pi(z+d) / h)$

Defined in $-d \leq z \leq-(d-h)$. The Fourier coefficients $C_{n m}$ 's are obtained from

$C_{n m}=\frac{2}{h} \int_{-d}^{-(d-h)} \varsigma_{n}^{j}(a, z) \cos (m \pi(z+d) / h) d z$

In addition to the boundary condition, the exterior and beneath potentials must be matched to ensure continuity at the interface between the exterior and beneath regions. This results in conditions on the potential,

$\Phi_{1}^{j}=\varsigma_{n}^{j} \quad$ on $\quad r=a,-d \leq z \leq-(d-h)$

By applying the boundary and matching conditions, we arrive at the key equations,

$C_{n m}=\frac{4}{h} \frac{i A_{n}^{j}}{\pi k a H_{n}^{\prime}\left(k a_{j}\right)}\left[\frac{h^{2} k \sinh k h(-1)^{n}}{\left(n^{2} \pi^{2}+h^{2} k^{2}\right) \cosh k d}\right]$

Based on the derived velocity potentials, the wave excitation forces can be computed. The exciting forces on the each cylinder are obtained by the integration of the pressure on the surface of the respective cylinder. Surge force given by the real part of $F_{x} e^{-i \omega t}$ is obtained as follows,

$$
\begin{aligned}
& F_{x}=-i \rho g H \int_{0}^{2 \pi} \int_{-(d-h)}^{0} \sum_{n=-\infty}^{\infty} \Phi_{1}^{j}(r, z) e^{i n \theta} a \cos \theta d \theta d z \\
& F_{x}=-\frac{i \rho g H}{k^{2} H_{1}^{\prime}\left(k a_{j}\right)} \frac{[\sinh k d-\sinh k h]}{\cosh k d}\left(A_{-1}^{j}-A_{1}^{j}\right)
\end{aligned}
$$

Sway force given by the real part of $F_{y} e^{-i \omega t}$ is obtained as follows,

$$
\begin{aligned}
& F_{y}=-i \rho g H \int_{0}^{2 \pi} \int_{-(d-h)}^{0} \sum_{n=-\infty}^{\infty} \Phi_{1}^{j}(r, z) e^{i n \theta} a \sin \theta d \theta d z \\
& F_{y}=-\frac{i \rho g H}{k^{2} H_{1}^{\prime}\left(k a_{j}\right)} \frac{[\sinh k d-\sinh k h]}{\cosh k d}\left(A_{-1}^{j}+A_{1}^{j}\right)
\end{aligned}
$$

Heave force given by the real part of $F_{z} e^{-i \omega t}$ is derived as follows by integrating the potential around the cylinder, 


$$
\begin{aligned}
& F_{z}=i \rho g H \int_{0}^{2 \pi} \int_{0}^{a} \sum_{n=-\infty}^{\infty} \varphi_{b}^{j}(r, z) e^{i n \theta} r d r d \theta \\
& F_{x}=2 i \pi \rho g H\left[\frac{C_{00}}{2} \frac{a^{2}}{2}+\sum_{m=1}^{\infty} C_{0 m} \frac{I_{1}(m \pi a / h)}{I_{0}(m \pi a / h)} \frac{h a}{m \pi}(-1)^{m}\right]
\end{aligned}
$$

The free-surface elevation in general is given by

$\eta(x, y)=\frac{H}{2} \Phi(x, y)$

In particular, the free-surface elevation outside the cylinders can be calculated by substituting equation (7) into the above equation,

$\eta(x, y)=\frac{H}{2}\left[e^{i k r \cos (\theta-\beta}+\sum_{j=1}^{N} \sum_{n=-\infty}^{\infty} A_{n}^{j} Z_{n}^{j} H_{n}\left(k r_{j}\right) e^{i n \theta_{j}}\right]$

The wave run-up on the outer surface of the $j$ th cylinder is given by,

$\eta_{1}^{j}\left(a_{j}, \theta_{j}\right)=\frac{H}{2}\left[\sum_{n=-\infty}^{\infty} \frac{-2 A_{n}^{j}}{k \pi a_{j} H_{n}^{\prime}\left(k a_{j}\right)}\left\{i+\frac{G J_{n}\left(k a_{j}\right)}{J_{n}^{\prime}\left(k a_{j}\right)}\right\} e^{i n \theta_{j}}\right]$

For the interior region of the $j$ th cylinder, the free-surface elevation can be calculated from equation (14) as given below,

$\eta_{2}^{j}(x, y)=\frac{H}{2}\left[\sum_{n=-\infty}^{\infty} B_{n}^{j} J_{n}\left(k r_{j}\right) e^{i n \theta_{j}}\right]$

\section{RESULTS AND DISCUSSION}

Firstly, the wave excitation forces evaluated by the developed numerical analysis in the present study are examined to validate the availability with some numerical results obtained from HOBEM (Choi et al., 2000).

Fig. 2 shows the comparison of total wave forces acting on the impermeable single cylinder for different incident wave angle $(\beta)$. In these figures, the wave excitation forces are non-dimensionlized by $\rho g \mathrm{Ha}^{2}$ and the abscissa denotes the non-dimensional wave number $(k a)$. The present results are in good agreement with the results obtained from HOBEM. It is shown that the surge forces decrease as the incident wave angle increase and the sway forces present converse pattern to the case of surge forces. The heave forces, however, have same values for all cases. It is indicated that the heave forces are not influenced by the variation of incident wave angle for the impermeable single cylinder.

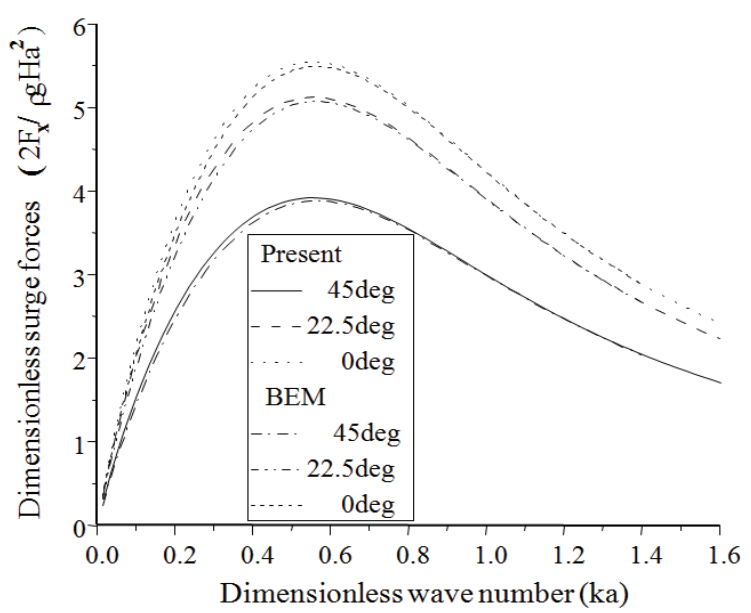

(a) Surge force.

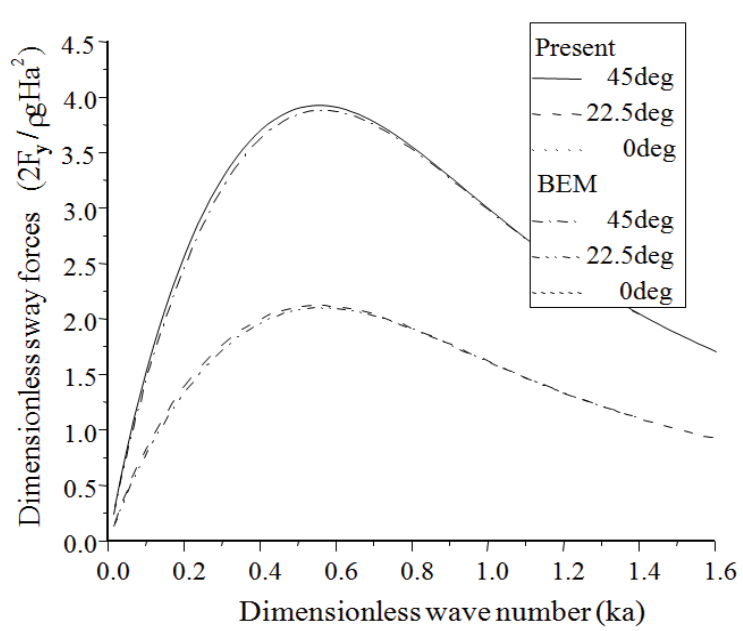

(b) Sway force.

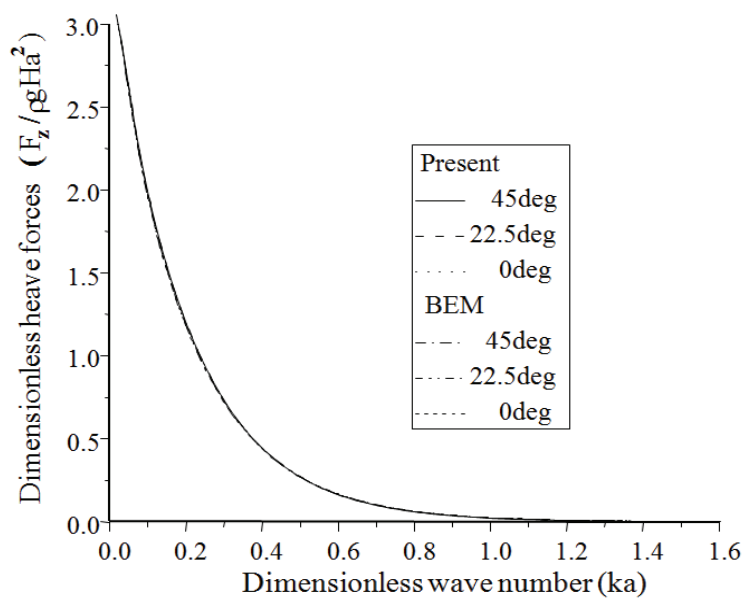

(c) Heave force.

Fig. 2 Non-dimensionless total wave force on the impermeable single cylinder with $a=8.44 \mathrm{~m}, d=200 \mathrm{~m}, h=165 \mathrm{~m}$, $G=0$ for different incident wave angle $(\beta)$. 


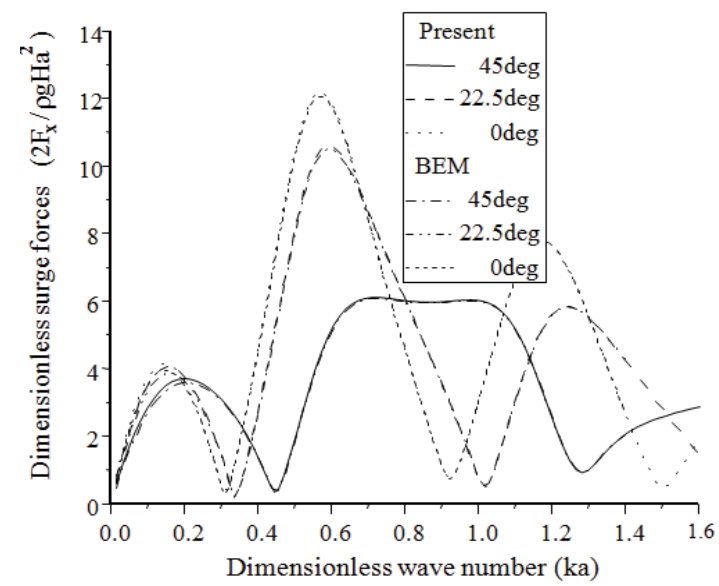

(a) Surge force.

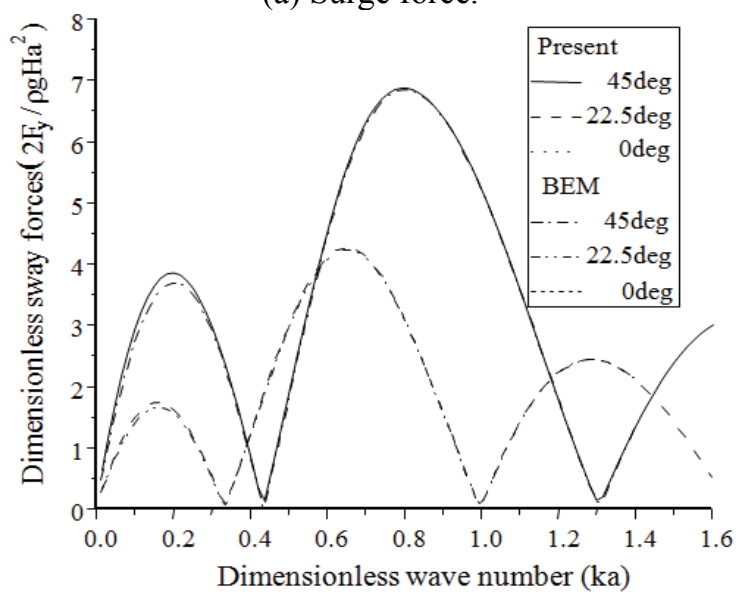

(b) Sway force.

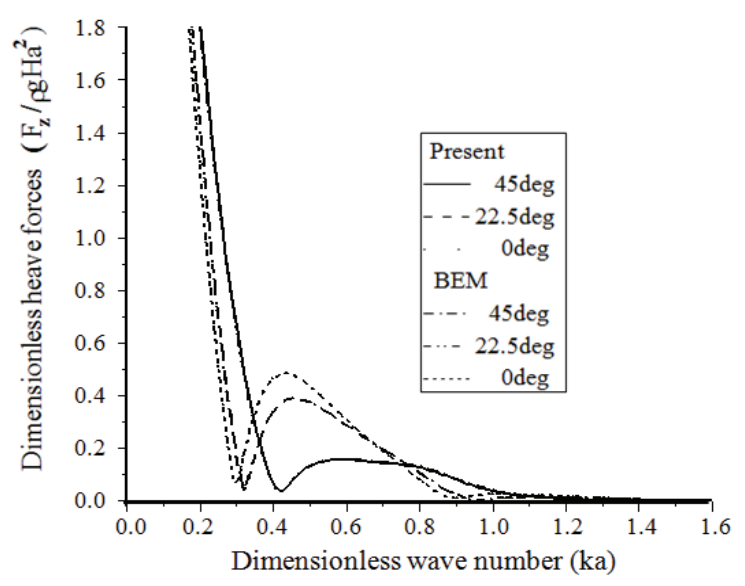

(c) Heave force.

Fig. 3 Non-dimensionless total wave force on the impermeable two cylinders with $a=8.44 m, d=200 m, h=165 m$, $R=86.25 \mathrm{~m}, G=0$ for different incident wave angle $(\beta)$.

Fig. 3 shows the non-dimensionless total wave forces on the impermeable two cylinders for different incident wave angle. The various parameter are $a=8.44 m, d=200 m$ and $h=165 \mathrm{~m}$. The cylinders are situated at $(0,-43.125) \mathrm{m}$ and $(0,43.125) \mathrm{m}$. The wave excitation forces are significantly influenced by the interaction effects between wave and structure and the incident wave angle play very important role on the interaction. It is understood that interaction effects can be remarkably important in determining the amplitude of the wave excitation forces. It is noted that the wave force by the present method gives the good agreement to the results from HOBEM. Therefore, the present method on wave excitation force evaluation is quite useful to evaluate the wave forces acting on the array of impermeable truncated vertical circular cylinders.

It was reported that the porous circular cylinder is significantly effective to reduce the hydrodynamic force and wave run-up on the cylinder compared to the impermeable cylinder (Williams and Li, 2000). Fig. 4 show the wave excitation forces on the permeable three cylinders for the different porosity rate $(G)$. The cylinders are situated at ($49.796,0) m,(24.898,43.125) m$ and $(24.898,-43.125) m$. In these figures, $G=0$ means an impermeable cylinder and the wave excitation forces are non-dimensionlized by $\rho g \mathrm{Ha}^{2}$.

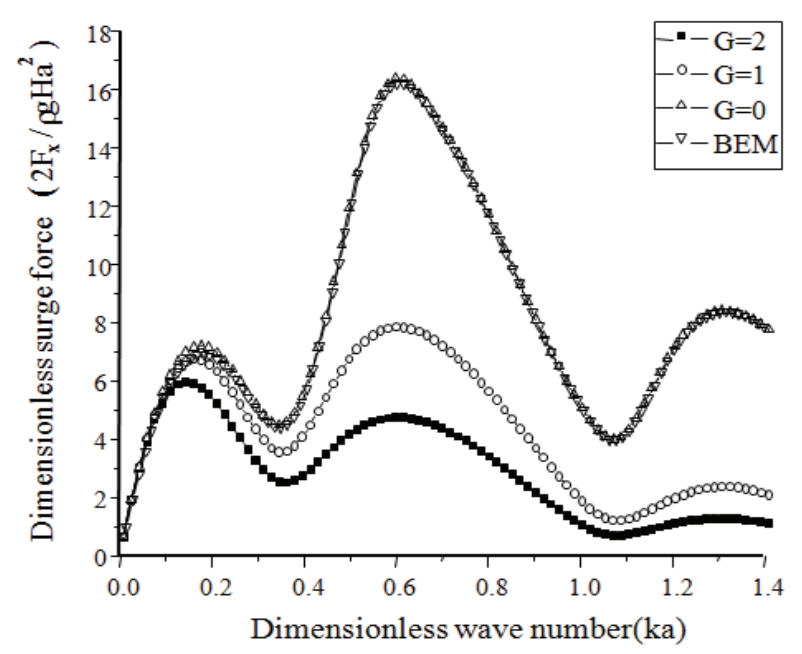

(a) Surge force.

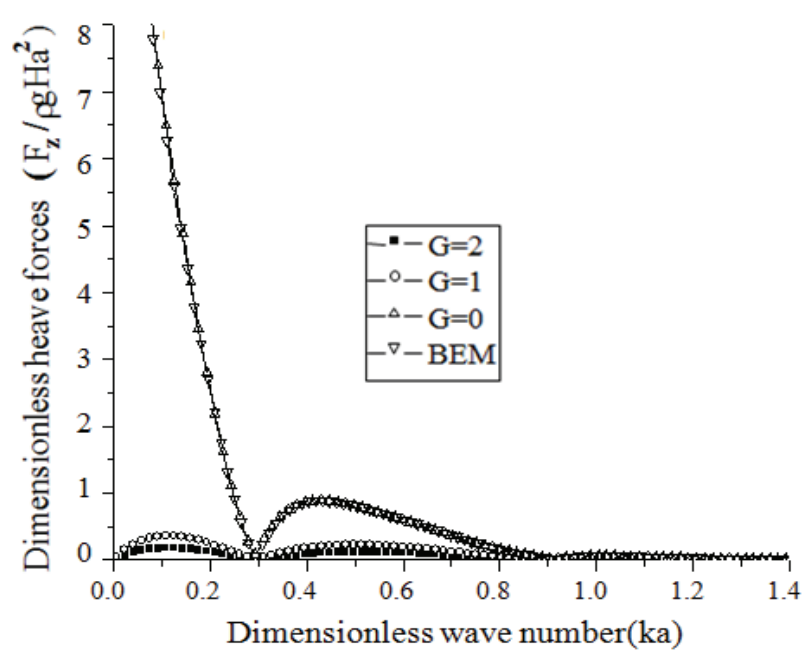

(b) Heave force.

Fig. 4 Non-dimensionless total wave force on the permeable three cylinders with $a=8.44 m, d=200 m, h=165 m, R=86.25 m$, $\beta=0^{0}$ for different porosity $\operatorname{rate}(\mathrm{G})$. 


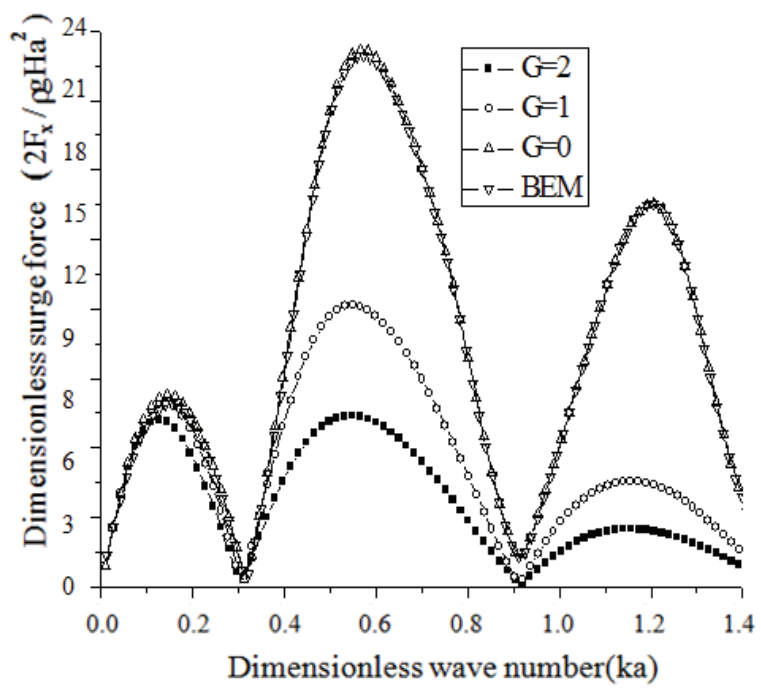

(a) Surge force.

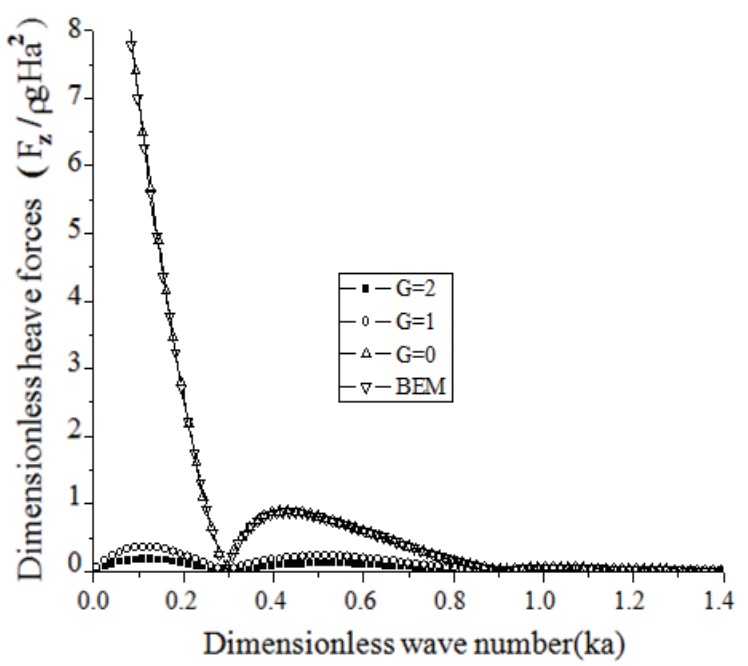

(b) Heave force.

Fig.5 Non-dimensionless total wave force on the permeable four cylinders with $a=8.44 m, d=200 m, h=165 m, R=86.25 m$, $\beta=0^{0}$ for different porosity rate $(\mathrm{G})$.

The abscissa denotes the non-dimensional wave number $(k a)$. The surge forces are slightly decreased as the porosity rate increase when the wave number is less than 0.4 , but their decreasing rate is dramatically increased when wave number is greater than 0.4 compared to fore cases. It is noted that the porous cylinders are very efficient on the reduction of surge forces as the incident wave becomes a short wave. The heave forces are remarkably decreased by the porosity rate for all wave numbers and the pattern of heave force is very similar to the case of a horizontally submerged plate disk. Therefore, the porous cylinders play very important role on the reduction of wave excitation forces, and especially it is significantly effective on the reduction of heave forces compared to other forces. When the porosity rate is zero, the present results are in good agreement with the results obtained from HOBEM.
To investigate the interaction effect between wave and structure more clearly, the non dimensionless total wave force on the permeable four cylinders is plotted in Fig. 5 and Fig. 6 for different porosity rate and incident wave angle.

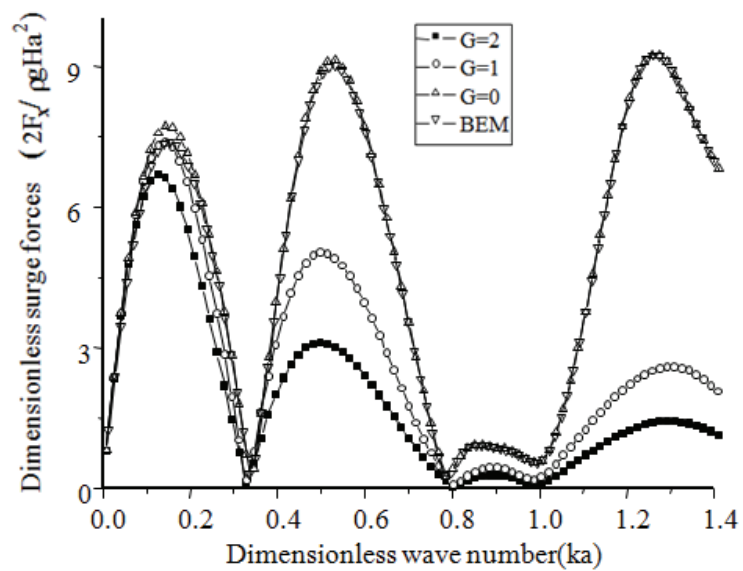

(a) Surge force.

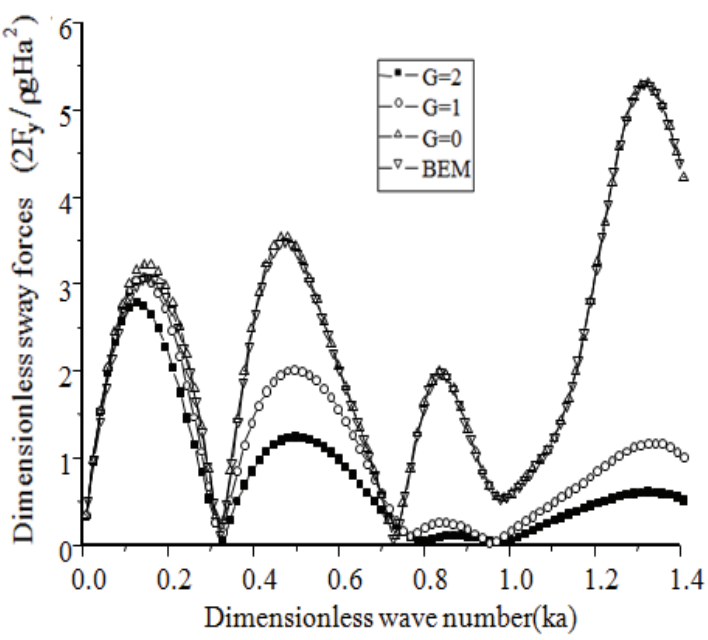

(b) Sway force.

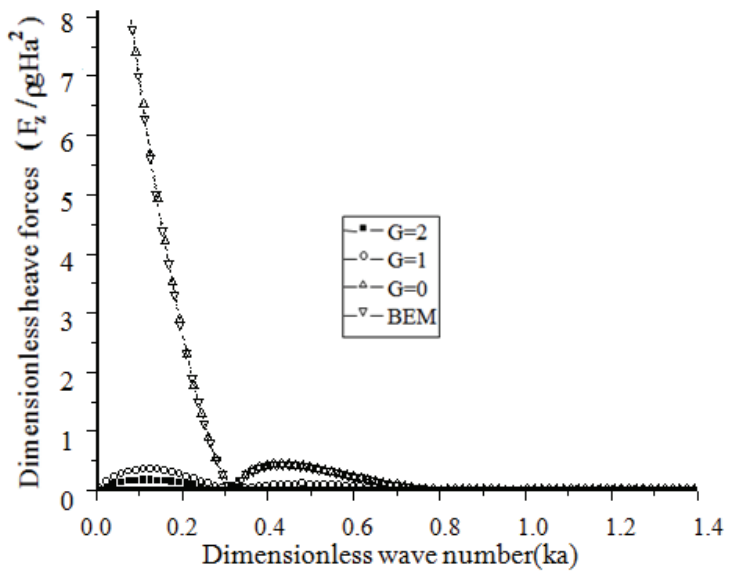

(c) Heave force.

Fig. 6 Non-dimensionless total wave force on the permeable four cylinders with $a=8.44 m, d=200 m, h=165 m, R=86.25 m$, $\beta=22.5^{\circ}$ for different porosity rate $(\mathrm{G})$. 
The cylinders are numbered clockwise from 1 to 4 , and are situated at $(-43.125,43.125) m,(43.125,43.125) m$, $(43.125,-43.125) m$ and $(-43.125,-43.125) m$ respectively. In these figures, the present results also give the good agreement to the results from HOBEM when the porosity rate is zero.It can be seen that the wave excitation forces are strongly influenced by the interaction effects caused by the variation of incident wave angle and when the incident wave angle is $22.5^{\circ}$, the interaction effects clearly show as the wave number increases compared to zero. As expected, the wave force on the cylinder can be reduced significantly by the porosity and the forces tend to decrease as the porosity parameter $G$ increases. In this case, the surge forces are also dramatically decreased by the porosity when the incident wave becomes a short wave compared to the cases of long wave and the heave forces are remarkably decreased by the porosity for all wave numbers.

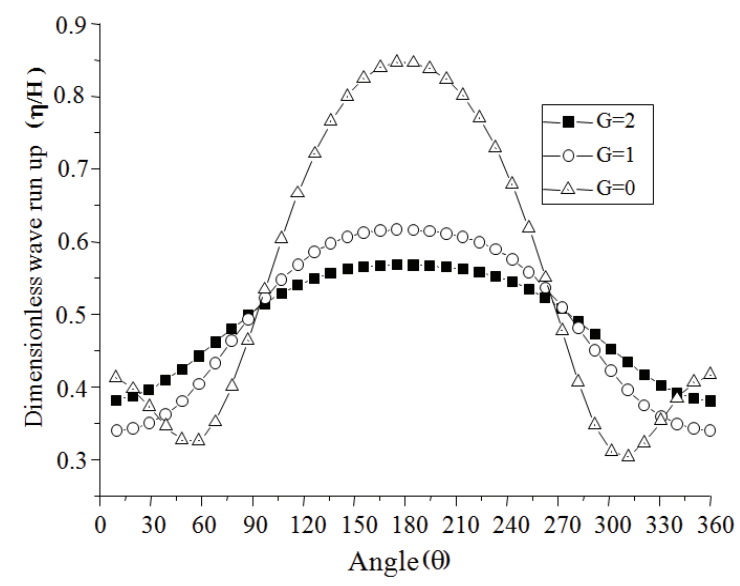

(a) Front cylinder.

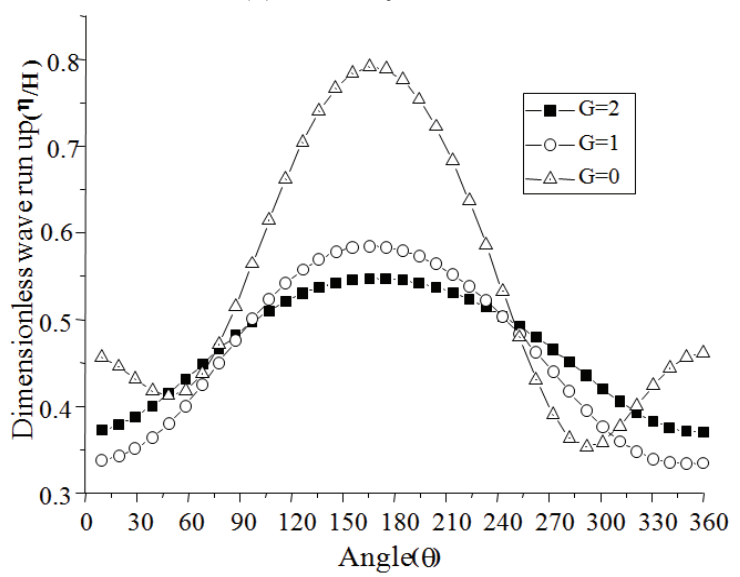

(b) Rear cylinder.

Fig.7 Non-dimensionless wave run up on the permeable four cylinders with $a=8.44 m, d=200 m, h=165 m, R=86.25 m, \beta=0^{0}$ for different porosity $\operatorname{rate}(\mathrm{G})$.

Fig. 7 shows the wave run-up amplitude both front and rear cylinder for $k a=0.61$. The maximum wave run-up occurs at $\theta=180^{\circ}$ and as porosity $G$ increase, the maximum wave run-up tends to decrease. Because the reflection wave from cylinders is rapidly decreased by the damping effect of energy that is produced as the incident wave goes through the permeable cylinders. It is noted that the porosity has the significant effect to reduce the wave run-up.

\section{CONCLUSIONS}

The interaction of water waves with arrays of truncated vertical circular cylinders has been investigated theoretically. Under the assumptions of potential flow and linear wave theory, a semi-analytical solution has been obtained by an eigenfunction expansion method based on Williams and $\mathrm{Li}$, 2000. Analytical expressions have been developed for the wave motion in the three regions i.e. a single exterior region, $N$ interior regions and $N$ beneath regions. Moreover, the present method can apply to an array of truncated vertical circular cylinders.Numerical results are compared with the results obtained by HOBEM to verify present method and have been presented which illustrate the effects of various incident wave angle and structural parameters such as impermeable or permeable cylinders on the wave excitation forces. It has been found that the present method on wave excitation force evaluation is quite useful to evaluate the wave forces such as surge, sway and heave forces acting on the array of truncated vertical circular cylinders and the porosity of the truncated vertical circular cylinders is very efficient on a significant reduction in both the wave excitation forces and wave run-up.

\section{ACKNOWLEDGEMENTS}

This research was supported by World Class University (WCU) program through the National Research Foundation of Korea, funded by the Ministry of Education, Science and Technology (R33-2009-000-10098-0).

\section{REFERENCES}

Abramowitz, M., Stegun, I.A., 1972. Handbook of Mathematical Functions, Dover Publications, New York.

Cho, I.H., 2003. Wave control by an array of N Bottommounted porous cylinders, Korean society of Coastal an Ocean Engineers, 15(4), pp. 232-241.

Choi, Y.R. and Hong, S.Y and Choi, H.S., 2000. An analysis of second-order wave forces on floating bodies by using a higher-order boundary element method. Journal of Ocean Engineering, 28, pp.117-138.

Isaacson, Michael de St. Q., 1977. Shallow wave diffraction around large cylinder, Journal of Waterway, Port, Coastal and Ocean Engineering, 103, pp. 69-82. 
Isaacson, Michael de St. Q., 1978. Wave run-up around large circular cylinder, Journal of Waterway, Port, Coastal and Ocean Engineering, 104, pp. 69-79.

Kagemoto, H. and Yue, D.K.P., 1986. Interactions among multiple three-dimensional bodies in water waves; an exact algebraic method, Journal of Fluid Mechanics, 166, pp. 189-209.

Linton. C.M. and Evans.D.V., 1990. The interaction of waves with arrays of vertical circular cylinder, Journal of Fluid Mechanics, pp. 549-569.

MacCamy, R.C. Fuchs, R.A., 1954. Wave forces on piles: a diffraction theory, Tech. Memo No. 69, US Army Corps of Engineers, Beach Erosion Board.

Mclver, P. and Evans, D.V., 1984. Approximation of wave forces on cylinder arrays, Applied Ocean Research, 6, pp. 101-107.

Spring, B.H. andMonkmeyer, P.L., 1974. Interaction of plane waves with vertical cylinders, Proceeding of the Fourteenth International Conference on Coastal Engineering, Copenhagen, Denmark, pp. 1828-1847.
Wang, K.H. and Ren, X., 1994. Wave interaction with a concentric porous cylinder system, Ocean Engineering, 21, pp. 343-360.

Williams, A.N. and Abul-Azm, A.G., 1989. Hydrodynamic interactions in floating cylinder arrays. Part II-Wave radiation, Ocean Engineering, pp. 217-264.

Williams, A.N. and Demirbilek, Z., 1988. Hydrodynamic interactions in floating cylinder arrays.Part I-Wave scattering, Ocean Engineering, pp. 549-582.

Williams, A.N. and Li, W., 1998. Wave interaction with a semi-porous cylindrical breakwater mounted on a storage tank, Ocean Engineering, pp.195-219.

Williams, A.N. and Li, W., 2000. Water wave interaction with an array of bottom-mounted surface-piercing porous cylinder, Ocean Engineering, pp.841-866.

Williams, A.N. and Rangappa, T., 1994. Approximate hydrodynamic analysis of multi-column ocean structures, Ocean Engineering, 21, pp. 519-573. 